\title{
Analysis of the International Competitiveness of Chinese Medicine Industry Based on the Diamond Model
}

\author{
Ying Liu, Yizhou Zhang \& Cong Xu \\ School of Economics and Management, North China Electric Power University, Beijing 102206, China \\ E-mail: zhang_yizhou@126.com
}

\begin{abstract}
The Chinese medicine industry always holds the balance in the Chinese medicine market of China. At present, the medicine industry has gradually formed the special industry system and group. By Porter's "Diamond Model" theory, the international competitive advantages and disadvantages of Chinese medicine industry are analyzed in this article, and corresponding suggestions about the industry development are provided.
\end{abstract}

Keywords: Chinese medicine, Diamond model, Competitiveness

Chinese medicine is the treasure of Chinese people, with the history of 5000 years. China is always has the name of "Kingdom of Chinese Medicine", and for a long time, there are about 130 countries in the world which import raw materials or patent medicines from China. According to the statistics of Chinese Ministry of Commerce, China has been the biggest country which produces and exports raw materials of medicines in the world, and China could produce about 1500 kinds of chemical medicines and middle medicines, and the total output has exceeded 8 ten thousand tons. Porter's diamond model is used in this article to analyze the international competitiveness of Chinese medicine industry of China, and the result shows that many challenges still exist when enhancing the competitiveness. Relative suggestions will be provided in this article.

\section{Diamond model}

In 1990, Michael Porter established a new analysis standard about the nation competitiveness in his book of "The Competitive Advantage of Nations", i.e. the "Diamond Model (Porter ME, 1990, P.64-117)". Porter's diamond model was composed by four basic decisive factors and 2 assistant factors (seen in Figure 1). Basic decisive factors include the production factor, the demand condition, the enterprise strategy, structure and competitiveness, and relative and supporting industries. Assistant factors are opportunity and government. These factors influence themselves each other, and compose the dynamic system of analyzing the industry competitive ability.

\section{Analysis of the international competitiveness of Chinese medicine industry}

\subsection{Production factors}

\subsubsection{Natural resources}

The Chinese medicine source of China is very abundant, and according to the statistics of Chinese medicine source survey up to data, the recorded Chinese medicine source amount in China has achieved 12807 (seen in Table 1). Only for 320 usual plant medicine materials, the total content achieves 8.5 million tons, and there are about 5000 kinds of Chinese herbal medicines which could be confirmed. There are over 700 production bases of Chinese medicines in China, and there are about 5 million mu lands to plant Chinese medicines (not including wood-medicines), and the output is about 0.4 million tons each year. Because of special geographical condition of China, the quality of medicines is very high, which could not been exceeded by other countries. At present, except a part of Chinese medicine materials are used to fulfill the domestic demands, quite part of medicine materials is exported. Relatively speaking, the medicine resources in foreign countries are deficient.

In recent years, because of excessive collection and the depravation of biological environment, many breeds of Chinese medicine are quickly reducing or disappearing, and in addition, the planting breeds begun to degenerate because of multiple plantings, and the quality and output of medicines all reduced (Zhu, 2000). According to relative statistics, in nearly 3000 rare and endangered plants, about $60 \%-70 \%$ of them has medicine values, and 168 sorts of plants have been listed in the List of Rare and Endangered Plants under State Protection of China, and 162 sorts of medicine animals were listed in the List of Intense National Protection of Wild Animal of China, where, Linfang, black bear, red deer, Gejie, and other forty kinds of animal significantly reduced, and the resource of muskiness has reduced $70 \%$ than $1950 \mathrm{~s}$, and the endangered status of tiger bones and rhinoceros horn has significantly influence the market supply of nearly 30 kinds of animal medicines, which are all challenging the development of the Chinese medicine industry of China. 


\subsubsection{Knowledge resources}

At present, China has formed complete Chinese medicine theory system, and about 0.3 million prescriptions of Chinese medicines have been saved. For Chinese medicines, China has abundant products with proprietary intellectual property rights, and in the international market competition, the Chinese medicine industry has the potential advantage to form the proprietary intellectual property rights.

But for a long time, China had not emphasized the intellectual property rights of Chinese medicine, and many knowledge fortunes of Chinese medicine have been lost, and in addition, the current patent system was established to protect chemical medicines by developed countries, and many domains about Chinese medicine could not be protected. For example, some ways of diagnosis such as acupuncture, Qigong, looking, listening, questioning, and feeling the pulse all could not be protected by the Patent Law. And the "herbal medicine" defined by the WHO only included raw materials of plant, and the Patent Law lacked in concrete operation rules for drinking dose, frying dose, and soup dose. On the one hand, developed countries utilized abundant experiences in the intellectual property rights to contest the intellectual property rights of Chinese medicine with China in the name of cooperation and development. On the other hand, they used the intellectual property rights as the weapon to monopolize the fortune of some intellectual property rights of Chinese medicine.

\subsubsection{Development of science and technology}

At present, most Chinese medicine production enterprises in China are middle and small enterprises which are difficult to realize the production of Chinese medicines with high efficiency and quality, but will waste the resources which have not been utilized reasonably. These enterprises which consume the resource of medicine materials to survive would not pay attention to science and technology, and become the principal part to support the technical innovation of the Chinese medicine research. Many researches and developments either repeat past researches in lower level, or could not form medicines or commodities to bring benefits.

The medicine industry is an industry with technical innovation. In recent years, many developed countries such as US, Japan, and Korea all strengthened the development and investment of traditional medicines, and their scientific research and production have entered into the orbit of modernization. But the Chinese medicine enterprises and academies of China still lack in sufficient investment of R\&D, especially for the research of drug nature, the confirmation and abstraction of effective component, and the alteration of new doses. According to the statistics of US PHRMA, in 2003, the R\&D charge about medicine in this association achieved 33 billion dollars, $15.6 \%$ of the sales, but this proportion in China was only about $1 \%$. In foreign countries, one medicine scientific and research person's charge is about from 125 thousand dollar to 226 thousand dollar each year, but in China, only 5000 dollar to 6000 dollar could be ensured. Low-level R\&D investment makes enterprise lack in the extension of new products, and with the competition of foreign chemical medicines and biological medicines, the market share of Chinese medicines could not increase continually. In addition, for the innovation of dose medicine, the traditional doses only include pill, pulvis, electuary, sublimed preparation, and decoction, but good breeds with quick, long, and high efficiency, small toxicity, side-effect, and dosage, and convenient storage, carrying, and taking are still rare, which is far from the popular tendency of international dose forms.

\subsubsection{Human resources}

Talents are the first condition of the R\&D of medicines. Deficient professional talents and weak innovational ability are common problems in the management layer of Chinese medicine enterprises in China. Though up to 2000, there were 30 Chinese medicine colleges and schools, and 14 undergraduate and specialized specialties, and 37000 students, 51 secondary Chinese medicine schools and 29000 students (Zhang, 2007), but comparing with the whole medicine market, the professional talents are still deficient, especially those talents with high and new scientific technologies and innovational spirit, and many pharmacy enterprises have deficient strengths and less R\&D investments, and the cultivation of talents are limited, and the innovational ability is weak. At the same time, most Chinese medicine enterprises lack in complete sales network and trained sales group. Above factors largely restrain the development of Chinese medicine industry of China, and seriously influence the production and sale of Chinese medicine (Chen, 2001, P.1-4).

Furthermore, in some specialties, the talents are so deficient, for example, for the medicine plant cultivation and relative subjects, the scientific technology staffs are reducing gradually in various scientific research academies of China, which is directly influencing the research of GAP, and becoming an obstacle in the course of Chinese medicine modernization.

\subsection{Demand conditions}

\subsubsection{International market}

The extension of the international market demand has provided extensive stage and space for the development of 
the Chinese medicine industry of China. The products of Chinese medicine of China are increasing stably, and many products have exported to 135 countries and regions, and parts of them have entered into the market through the enrollment of international drugs. In 2007, the imports and exports of Chinese medicine products of China achieved 1.18 billion dollar, which was the top year in the history.

But, because of different histories and cultural backgrounds, the western medical theory is different with Chinese medical theory in principle and thinking method. This difference between western culture and eastern culture brings "cultural bulwark" for the internationalization of Chinese medicine which could not be eliminated. At present, Chinese medicine is only legal in a few countries such as Australia and Singapore, and it is still rejected by most European and American countries from the normal medicine, and it lacks in legal status, and only exists by the mode of substitute medicine. And if it enters into these markets by the health-food supplement or the food additive, there are many strict limitations such as heavy metal content, pesticide residuals, toxicity component, and except for many components such as $\mathrm{Hg}$, arsenic, and lead, it could not be marked by the adaptability and medical range of medicines, and it could not be sold in the drugstore, which all will influence the Chinese medicine industry to enhance its international competitiveness.

\subsubsection{Domestic market}

The quick growth of Chinese population promotes the increase of the drug demand, and from 2003, China had entered into the "old age", and large old group established the base for the extension of the drug market. The statistical data indicated that there were $72 \%$ Chinese consumers who could accept Chinese medicines, and this proportion would be higher in rural regions and old group. In the future, with the development of economy, the enhancement of people's life level and self-health protection, the development space of the drug market could be enlarged further. In Porter's national competitive advantage theory, quickly growing domestic demand could strengthen the national competitiveness of the industry, and could encourage manufacturers to introduce science and technology, renovate facilities, build larger and more efficiently workshops (Shi, 2002).

In Jan to May of 2007, the medicine manufacture had completed 214.624 billion Yuan of sale together, where, the sales amounts of the drink-pill of Chinese medicine and the Chinese medicines respectively were 9.342 billion Yuan and 47.661 billion Yuan, comparatively increasing $32.91 \%$ and $19.69 \%$, and the whole sale of the industry could keep the quick growth level. The investment circle of some industries in China universally emphasized the industry of Chinese medicine, and many listed companies take the investment of Chinese medicine as the new direction of transformation and extension, and many investments from tobacco, real estate, and daily health product are flowing to the domain of Chinese medicine. Shanghai JAHWA United Co. Ltd, Shanghai Fortune Industrial Co. Ltd, Green-valley Group, successively invested in the industry of Chinese medicine, and Thunis, Haier, and many enterprises begin to extend to the domain of Chinese medicine. Many chemical pharmacy manufacturers such as Shijiazhuang Pharmaceutical Co. Ltd and Shenzhen Neptunus Bioengineering Holodings Co. Ltd also begin to invest and develop Chinese medicines.

\subsection{Relative and supporting industries}

The industry chain of Chinese medicine industry is very long, and it covers many industries, and the upstream industry if the R\&D of new Chinese medicines, the mid-stream industry if the production of Chinese medicine, and the low-stream industry is the circulation of Chinese medicine, which all compose the main chain of the Chinese industry chain. In addition, many suited service systems such as traffic, wrapper, pharmacy machines, and Chinese medicine information consultation also could provide relative services for the development of the Chinese medicine industry, and exert important promotion function.

The production of the Chinese medicinal materials is the key part for the R\&D of new Chinese medicine, the clinic application, the production of Chinese preparation, and the foreign and domestic trade and its competitiveness will directly influence the international competitiveness of the Chinese medicine industry. Most upstream industries adopt the mode combining the Chinese medicine manufacturing enterprises with the medicinal materials supply source, and most large medicinal materials bases adopt the mode of cooperation contract, and enterprises would assume main market risk, so farmers' risk will be reduced, and the large fluctuation of the medicinal materials price could be avoided. The distribution and service of the low-stream industry are the key part, and its organization mode is the lever to win for Chinese medicine enterprises. Therefore, many Chinese medicine enterprises of China have paid more attention to the low-stream industry of the operation flow, i.e. the marketing stage extends to the upstream industry, and established powerful marketing network to win in the market competition.

\subsection{Enterprise strategies, structure and competitiveness}

\subsubsection{Organization and management of enterprises}

Chinese medicine production enterprises of China are numerous, dispersive, and small, and the research and 
development of the international market is far deficient, and many enterprises could not actively invest in the innovational of technology and products, which is largely different with foreign pharmacy enterprises ( $\mathrm{Li}, 2003$, P.48-51).

To implement modernization management, Chinese medicine enterprises of China should compete in the international market, and the standardization of Chinese medicine is the basic and first condition for the modernization and internationalization of Chinese medicine. In many years' management practice, European and American developed countries have established a set of complete, strict, and standard quality control standard including GAP, GCP, GSP, GLP, and GMP. But at present, the standardization of Chinese medicine in China is only in the start. For the source of Chinese medicine, the Chinese medicinal materials planting management criterion was issued in 2002, and it has not been implemented in the whole China. For the making and abstracting technology of Chinese herbal medicines, the standards of only about 70 kinds of Chinese drink medicine have been accomplished, and the abstracting technique of Chinese medicinal materials are not standard, and the control of the product quality has not been perfected. For the quality test of Chinese medicines, most tests are still only in the level of determining the nature and testing individual components. For the evaluation of drug nature, drug effect, and toxicity of Chinese medicines, the domestic GCP and GLP have not been implemented for a long time, and the mutual unity with international current standards only begins, and various experiment data have not been recognized by the international society. It is obvious that China needs to use standardization to promote the modernization of Chinese medicine, and enhance the international competitiveness.

\subsubsection{Competitive opponents}

Because of the attraction of the development foreground of Chinese medicine industry, many countries such as southeast Asia, Korea, and Japan have adopt modern technology to research Chinese medicines and occupy the international market of Chinese herbal medicine. In the future, the Chinese medicine industry competitors of China will come from three regions (Guo, 2003, P.97-100).

The first regions is US which is the largest country consuming the plant medicines in the world. At present, GNC and other companies have not only international resources, production and development ability, but also large plant medicine groups with sales network. the second region is western Europe, and the countries in this region have the history to use and produce plant medicines, and the enterprises in these countries have large capitals, product development ability and advanced production technology, and they have powerful competitiveness in the international market of herbal medicine. The third region includes Japan and Korea. Their Chinese medicines will impact the Chinese market with the decrease of the custom of Chinese medicine, and their perfect market operation mechanism, marketing strategy, management experience, and advanced technology, will also bring large threats to Chinese medicine enterprises. The yearly total production value of Japanese Chinese medicines has exceeded 100 billion Yen, and many Chinese medicines such as Jiu Xin Wan have successfully occupied the international market. At the same time, Japan is building the base of herbal medicinal materials, and has established large plan of Chinese medicinal materials development. About 100 medicinal materials in the Korean market could be produced in the Korea, and many of them could export to other countries. Only for the ginseng, the sale could achieve 0.2 billion dollar each year for Korea, which is tens of times than Chinese ginseng.

\subsection{Opportunities}

The first opportunity is the economic globalization. Chinese medicinal materials are the traditional export products of China, and after China joined into WTO, China could attract more foreign capitals, technologies and management experiences, change and enhance traditional industry of Chinese medicinal materials. And the quick enterprise differentiation will create good opportunity for the sale and intensive development of Chinese medicine industry. After China joined into the multilateral trade system, the export environment of Chinese medicinal materials has been largely widened. Many principles such as the most-favored-nation clause, the market admittance, the elimination of "trade bulwark" are also very rare chances for the development of the Chinese medicine industry.

The second opportunity is the tide of returning to the nature and the new demand of healthy. Quick life mode induces many psychological diseases, and the modern medical modern has turned from simple disease treatment to the mode integrating prevention, health protection, treatment, and healing (Me, 2003). In the influence of "returning to the nature", people begin turning to the nature medicinal materials for the prevention and treatment of diseases, and the Chinese herbal medicines with long clinic history. The market capacity of corresponding products of plant raw materials is increasing largely.

The third opportunity is the development of modern science. In recent 20 years, a series of chemical separation 
and analysis identification technologies such as molecule biology, biological engineering technology, and micro-electronics technology, and various new filtering technologies have largely strengthened the R\&D ability of chemical medicines, and the invention of a series of medicines with high efficiency and minimum content has brought revolutionary change for the research of chemical medicines. These new biological technologies could be used to enhance the effect and veracity of the Chinese medicine treatment, and confirm the gene treatment method of Chinese medicine.

\subsection{Government}

The government is the factor with important influence. Chinese government has highly emphasized the development of Chinese medicine industry all along, and largely supported this industry. As the main content, the Chinese medicine industry has been listed in the "Eleventh Five-year Plan", and the modernization of Chinese medicine has been one of important contents of high technology industry. "Developing Chinese herbal medicines and biological medicines" has also been listed in the key works of the strategy of develop-the-west. In addition, to support the R\&D of new medicines, in the period of "the Ninth Five-year Plan", China had established the new medicine selection center, the drug safety evaluation center, and the new medicine clinic base according to international standard. Various local governments also started from the advantages of local resources, and largely develop the industries with special characteristics. At present, the modern Chinese medicine industry chain of China has gradually gone to perfection, and the scale and benefit of Chinese medicine industry enterprise are being enhanced continually. These aspects are all related with the industry support policies of the government.

Of course, the Chinese medicine industry is also limited by many governmental policies. Aiming at various parts of the Chinese medicine industry chain, the government has constituted a serious of standard and criterion, such as GAP, GEP, GMP, GCP, GLP, and GSP. These policies put forward higher requests for the Chinese medicine industry, and all Chinese medicine enterprises should actively face the challenge, and continually enhance their own qualities.

\section{Conclusions}

According to above analyses, following challenges should be emphasized when enhancing the international competitiveness of Chinese medicine industry, (1) incomplete quality standard of Chinese medicine product, (2) different cultural backgrounds, (3) lower technical innovational ability, (4) weak intellectual property rights production, (5) serious technical trade bulwark limitation, (6) Japanese and Korean relative advantage which has been formed in the global natural medicine market, (7) the large impact of foreign Chinese medicines in the domestic market, (8) the problem about the sustainable utilization of Chinese medicine resource.

To enhance the international competitiveness of Chinese medicine industry, following points should be concerned, (1) paying attention to developing the senior breeds and strengthening the standardization and good quality, and building more famous brands, (2) increasing the R\&D investment of Chinese medicines and adjusting the planting breeds of Chinese medicines, and enhancing the additive values of products, (3) extending the application range of Chinese medicines and enhance the comprehensive utilization value, (4) strengthening the marketing power and perfecting the circulation channel of medicinal materials, (5) integrating the resources of Chinese medicine industry when necessary, and regrouping Chinese medical enterprises, (6) starting from trademark protection and strengthening the protection of the intellectual property rights of Chinese medicine, (7) perfecting relative supporting industries and suited establishments, (8) strengthening the international communication and development of Chinese medicine culture, and perfecting the standard system which could not only accord with the characteristics of Chinese medicine, but also be accepted by the world.

\section{References}

Chen, Chong. (2001). Discussion about the Strategy of China Modernization. World Notes Plant Medicine. No.16(1). P.1-4.

Guo, Zhixin, Zhao, Libin \& Jiang, Jianlan. (2003). Current Situation and Strategy of Chinese Materia Medica Internationalization. Chinese Traditional and Herbal Drugs. No.34(2). P.97-100.

Li, Guoju. (2003). The Modernization Research of Chinese Medicine and the Modernization of Chinese Medicine Enterprises. Pharmacology and Clinics of Chinese Materia Medica. No.19(1). P.48-51.

Me, Li. (2003). Analysis of Chinese and Foreign Chinese Medicine Markets. Beijing: China Medical Technology Press.

Porter ME. (1990). The Competitive Advantage of Nations. New York: Free Press. P.64-117. 
Shi, Jianyong. (2002). Chinese Medicine Industry Economy and Development. Shanghai: Shanghai Scientific and Technical Publishers.

Zhang, Qing. (2007). Study on the Transforming of TCM Industry from Comparative Advantage to Competitive Advantage. Soft Science. No.3.

Zhu, Guoguang. (2000). How Chinese Medicines Enter into the Market of Europe. Beijing: China Medical Technology Press.

Table 1. Statistics of Chinese medicine resources in China

\begin{tabular}{|c|c|c|}
\hline Sort & Kind & Amount \\
\hline \multirow{3}{*}{ According to sources } & Plant & 11145 \\
\cline { 2 - 3 } & Animal & 1586 \\
\cline { 2 - 3 } & Mineral & 80 \\
\hline \multirow{3}{*}{ According to uses } & Chinese Traditional Medicines & 1200 \\
\cline { 2 - 3 } & National Medicines & 4000 \\
\cline { 2 - 3 } & Civil Medicines & 7000 \\
\hline
\end{tabular}

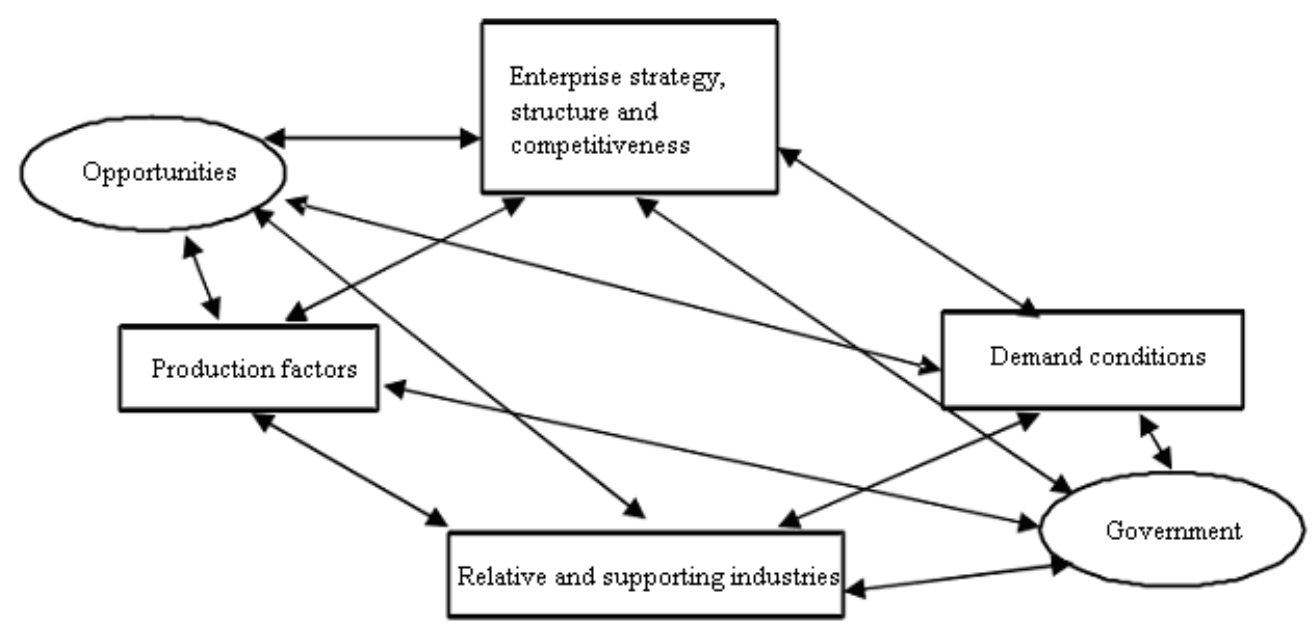

Figure 1. Porter's Diamond Model 